\title{
Use of Proliferation Signal Inhibitors in Cardiac Transplantation
}

\author{
Howard J. Eisen
}

Published online: 12 October 2014

(C) Springer International Publishing AG 2014

\begin{abstract}
Cardiac transplantation remains the definitive and most highly successful therapy for patients with advanced heart failure who have failed all other therapies. Long-term survival is limited by cardiac allograft vasculopathy, the transplant coronary artery disease, and malignancy. Standard, traditional immunosuppressive agents such as calcineurin inhibitors, anti-proliferative agents, and corticosteroids do little to attenuate cardiac allograft vasculopathy, which, because of its diffuse nature, is usually not amenable to percutaneous or surgical revascularization approaches, and may potentiate the developments of malignancies after cardiac transplantation. Proliferation signal inhibitors block the mammalian target of rapamycin, a critical protein involved in cell proliferation, and inhibit lymphocyte and smooth muscle cell proliferation. This results in inhibition of the alloimmune response and acute cellular rejection, even in the setting of low calcineurin levels. More significantly, they have been shown in several clinical trials to attenuate the progression of cardiac allograft vasculopathy when compared to mycophenolate or azathioprine regimens.
\end{abstract}

Keywords Proliferation signal inhibitors $\cdot \mathrm{m}$-TOR inhibitors · Immunosuppression - Cardiac transplantation - Acute cellular rejection · Cardiac allograft vasculopathy · Leukopenia . Hypertriglyceridemia $\cdot$ Calcineurin inhibitors $\cdot$ Vascular smooth muscle cells $\cdot$ Malignancies

\section{H. J. Eisen $(\bowtie)$}

Drexel University College of Medicine, 245 N. 15th Street,

Philadelphia, PA 19102, USA

e-mail: heisen@drexelmed.edu

\section{Introduction}

Cardiac transplantation is the oldest and most successful therapy for advanced heart failure with progressively improving survival over the past three decades. Most of the improvements have occurred in the first 6 months after transplant as a result of more effective therapies for treating or preventing acute cellular rejection and post-transplant infections [1]. Beyond the first year post-transplant, long-term survival continues to be limited by cardiac allograft vasculopathy (CAV) and malignancy, the leading causes of death in these patients (graft failure, listed as a separate cause of death, is usually caused by CAV; Fig. 1). Traditional immunosuppressive regimens using calcineurin inhibitors (CNI) such as cyclosporine or tacrolimus, anti-proliferative agents such as mycophenolate mofetil or azathioprine, and corticosteroids do not mitigate the development or progression of CAV and may potentiate the development of malignancies in these patients. Statins improve survival and reduce the severity of CAV and are the first therapy to improve definitively post-transplant outcomes $[2,3]$. Despite their widespread use in post-cardiac transplant management and the decreasing frequency of acute cellular rejection, CAV remains a major hurdle to long-term survival post-transplant.

\section{Pre-Clinical Studies of Proliferation Signal Inhibitors (PSIs)}

The mammalian target of rapamycin (mTOR) is a serine/ threonine protein kinase, which plays a critical role in proliferation in a variety of cell types. Disruption of the mTOR pathway can contribute to the development of malignancies as a result of loss of inhibition of proliferation [4]. Rapamycin or sirolimus inhibits mTOR by binding the cytosolic protein FKbinding protein 12 (FKBP-12) as does tacrolimus, but while 
Fig. 1 Causes of death after cardiac transplantation from the International Society of Heart and Lung Transplantation 2013 Registry. Graft failure is usually caused by cardiac allograft vasculopathy (CAV) (from Lund LH, Edwards LB, Kucheryavaya AY, et al. The Registry of the International Society for Heart and Lung Transplantation: Thirtieth Official Adult Heart Transplant Report-2013; focus theme: age. International Society for Heart and Lung

Transplantation. J Heart Lung Transplant. 2013;32:951-64.)
Adult Heart Transplants Cumulative Incidence of Leading Causes of Death (Transplants: January 1994 - June 2011)

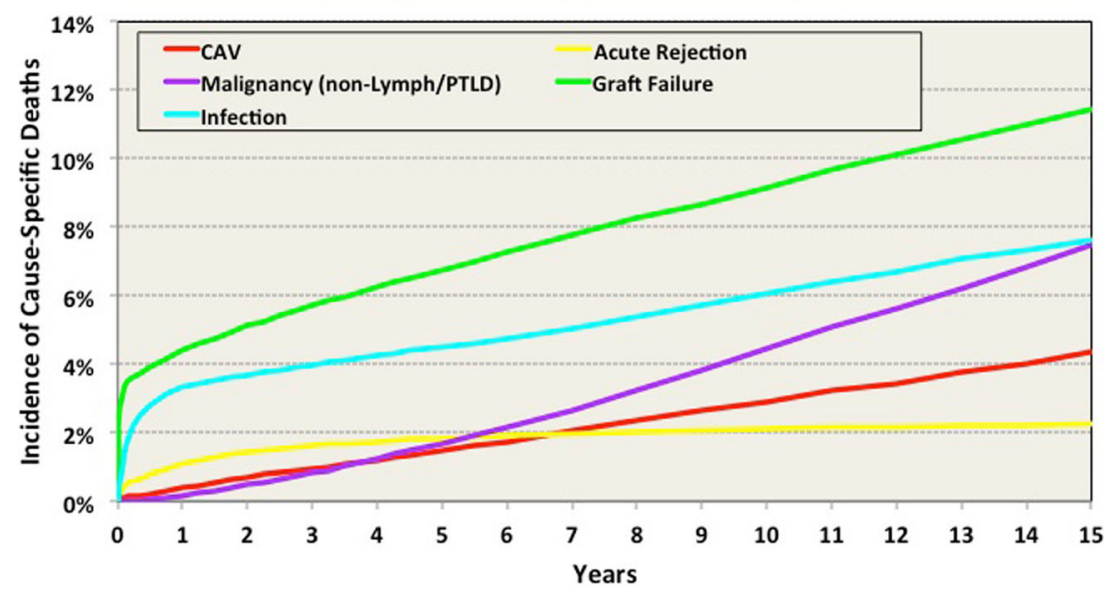

the tacrolimus-FKBP12 complex inhibits calcineurin with subsequent inhibition if interleukin 2, the sirolimus-FKBP12 complex binds to mTORC1, thus inhibiting mTOR [5] (Fig. 2). Sirolimus can inhibit proliferation of vascular smooth muscle cells and endothelial cells. PSIs have been shown to prevent rejection and CAV in rodent heterotopic heart transplant preparations and non-human primates $[6,7]$.

\section{PSIs and Interventional Cardiology}

Restenosis was common after angioplasty and stent deployment for coronary artery occlusions, occurring in up to $35-$ $40 \%$ of patients who underwent these procedures and manifested clinically by recurrent angina symptoms. Histologically and pathophysiologically, restenosis is characterized by smooth muscle cell proliferation, which is the vascular response to injury from the initial angioplasty/stent procedure. Inhibition of smooth muscle proliferation to inhibit this process was the rationale behind the development of drug eluting stents (DES), which would deliver PSIs directly to the site of stent delivery to blunt the proliferative injury response. The RAVEL study in Europe and the SIRIUS trial in the United States confirmed that sirolimus-eluting stents significantly reduced the rate of restenosis and clinical events after angioplasty and DES deployment compared to bare metal stents [8, 9]. Additional studies with newer generation stents eluting sirolimus or everolimus have confirmed these observations, although the anti-proliferative effects of these agents on endothelial proliferation inhibits re-endothelialization over the stent surfaces. Therefore, prolonged use of anti-platelet agents such as clopidogrel to prevent stent thrombosis is necessary $[10,11]$.

\section{PSIs Compared to Azathioprine In De Novo Cardiac Transplant Recipients}

Keogh and colleagues enrolled 136 cardiac transplant recipients in Australia and New Zealand and randomized them in an open-label manner to either $3 \mathrm{mg}$ or $5 \mathrm{mg}$ daily of sirolimus or azathioprine along with cyclosporine and corticosteroids [12]. At 6 months, the proportion of patients with an ISHLT Grade $3 \mathrm{~A}(2 \mathrm{R})$ rejection was $32.4 \%$ for sirolimus $3 \mathrm{mg}(P=0.027 \mathrm{vs}$. azathioprine), $32.8 \%$ for sirolimus $5 \mathrm{mg}(P=0.027)$, and $56.8 \%$ for azathioprine. Progression of CAV from week 6 to months 6 and 24 post-transplant was assessed by intravascular ultrasound (IVUS). Progression of CAV was significantly greater in the azathioprine group than in the two sirolimus groups. Serum creatinine was significantly elevated in the sirolimus $5 \mathrm{mg}$ group compared to the azathioprine group. Side effects more common in the sirolimus group included anemia, thrombocytopenia, diarrhea, hyperlipidemia, and mouth ulcerations.

Six hundred and thirty-four cardiac transplant recipients in Europe, North America, and Asia were enrolled in a randomized, double-blind study of everolimus $1.5 \mathrm{mg}$ daily $(0.75 \mathrm{mg}$ twice daily), $3.0 \mathrm{mg}$ daily ( $1.5 \mathrm{mg}$ twice daily), or azathioprine, all in combination with cyclosporine and corticosteroids [13]. The primary efficacy endpoint was the composite of biopsy-proven ISHLT Grade 3A (2R) rejection, rejection with hemodynamic compromise, death, graft loss or retransplantation, or loss to follow-up. At 6 months, the percentage of patients who met the primary efficacy endpoint was $27.0 \%$ for the $3.0 \mathrm{mg}$ everolimus group $(P<0.001$ compared to azathioprine), $36.4 \%$ for the $1.5 \mathrm{mg}$ daily everolimus group $(P<0.03)$, and $46.7 \%$ for the azathioprine group. This difference in the primary efficacy endpoint for the everolimus groups compared to azathioprine was accounted for mainly 


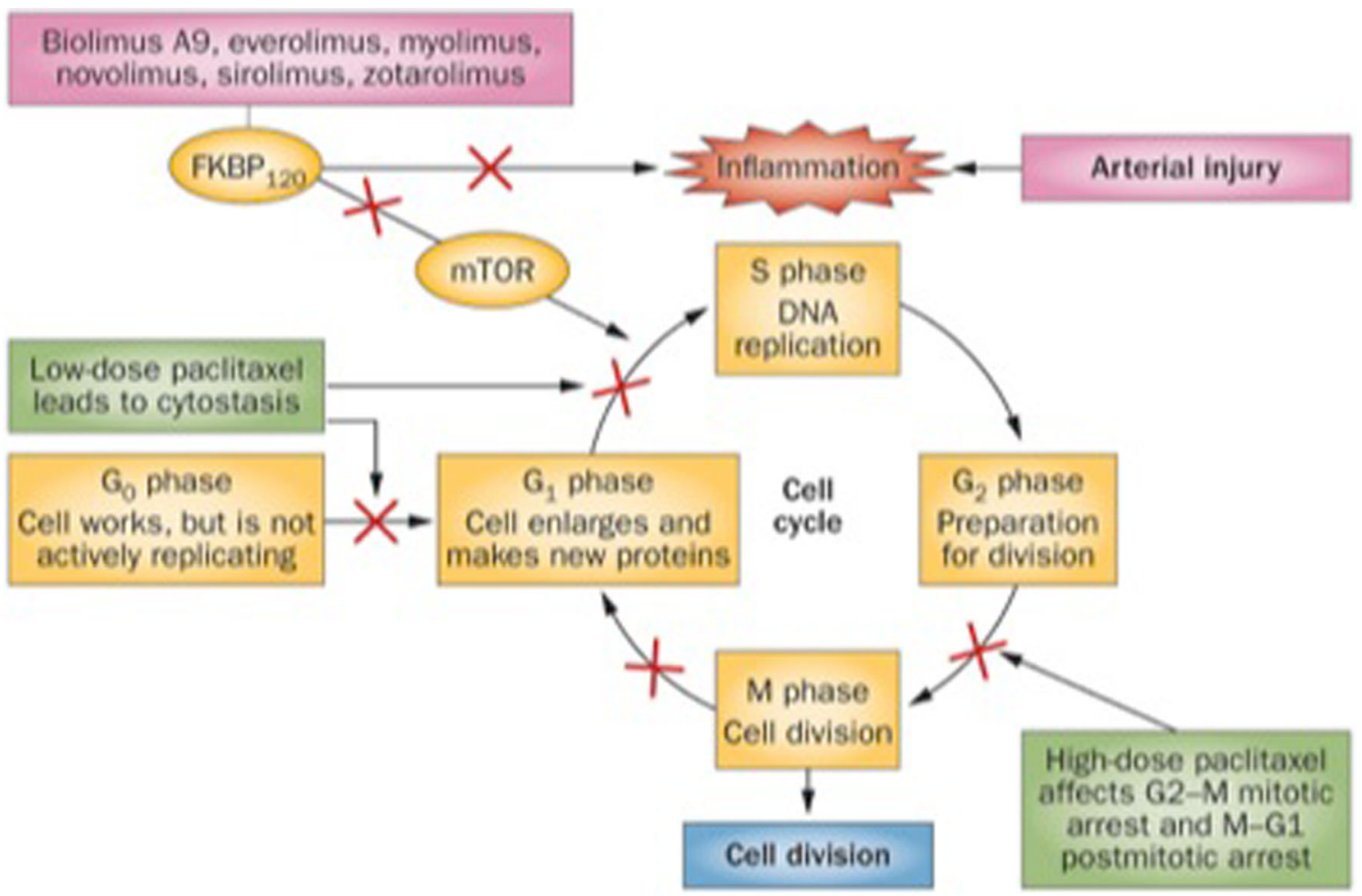

Fig. 2 Mechanism of action of Proliferation Signal Inhibitors or mTOR inhibitors (from Garg S, Bourantas C and Serruys PW, Nature Reviews Cardiology 2013;10:248-60)

by the reduction is the percentage of patients in the everolimus groups who had biopsy-proven ISHLT Grade 3A (2R) rejection with no differences in death or the other components of the primary efficacy endpoint. There was an IVUS sub-study to assess the progression of CAV. Paired IVUS studies were obtained at baseline (within several weeks post-transplant) and at 1 year post-transplant. The incidence of CAV was defined as a change in maximal intimal thickness (MIT) from the baseline to the 1 year post-transplant IVUS of $>0.5 \mathrm{~mm}$ in at least one matched slice (Fig. 3). The incidence of CAV was significantly lower for the two everolimus groups $(35.7 \%$ in the everolimus $1.5 \mathrm{mg}$ group, $P=0.045$ vs. azathioprine; $30.4 \%$ in the $3.0 \mathrm{mg}$ group, $P=0.01$ vs. azathioprine and $52.8 \%$ for the azathioprine group). The mean increase in MIT was reduced in the everolimus $1.5 \mathrm{mg}$ group $(0.04 \mathrm{~mm}, P=$ 0.01 vs. azathioprine; $0.03 \mathrm{~mm}$ in the $3.0 \mathrm{mg}$ group, $P=0.003$; and $0.10 \mathrm{~mm}$ in the azathioprine group). Other IVUS measures of CAV such as intimal cross-sectional area were all significantly reduced in the everolimus groups compared to the azathioprine group.

Serum creatinine was significantly elevated and glomerular filtration rate was significantly reduced in the two everolimus groups compared to the azathioprine group despite the fact that mean cyclosporine trough levels were similar in the three groups. Total cholesterol and triglyceride levels were elevated in the everolimus groups compared to the azathioprine group while the LDL and HDL levels were unchanged.
Thrombocytopenia was more common in the high dose everolimus group. Viral and specifically cytomegalovirus infections were significantly more common in the azathioprine group $(31.3 / 21.5 \%)$ than in the everolimus $1.5 \mathrm{mg}$ group (14.8/ $7.7 \% P<0.001$ vs. azathioprine) or the everolimus $3.0 \mathrm{mg}$ group $(17.1 / 7.6 \%, P<0.001)$. In contrast, bacterial infections were significantly more common in the everolimus $3.0 \mathrm{mg}$ daily group $(37.0 \%, P=0.001)$ than the azathioprine group $(24.8 \%)$ although this did not translate into increased mortality from sepsis or other bacterial causes.

An important observation from this study was that beyond 2 weeks after transplant, as long as the everolimus serum levels were therapeutic $(3-8 \mathrm{ng} / \mathrm{mL})$, the rates of cellular rejection were no different whether the trough cyclosporine levels were $50 \mathrm{ng} / \mathrm{mL}$ or up to $400 \mathrm{ng} / \mathrm{mL}$, demonstrating the important synergistic role that this PSI plays in conjunction with cyclosporine in preventing cellular rejection [14]. Another interesting observation was that non-fatal major adverse cardiac events (MACE) were reduced in the everolimus IVUS patients compared to the azathioprine IVUS patients and that the potential cost of care for MACE was higher in the azathioprine than in the everolimus group [15]. This study was limited by the fact that it was an ad hoc analysis.

The two PSI studies discussed above were the first in cardiac transplant recipients and, therefore, provided important insights into the effects of these drugs on mitigating CAV and CMV infections, but had significant limitations in that 


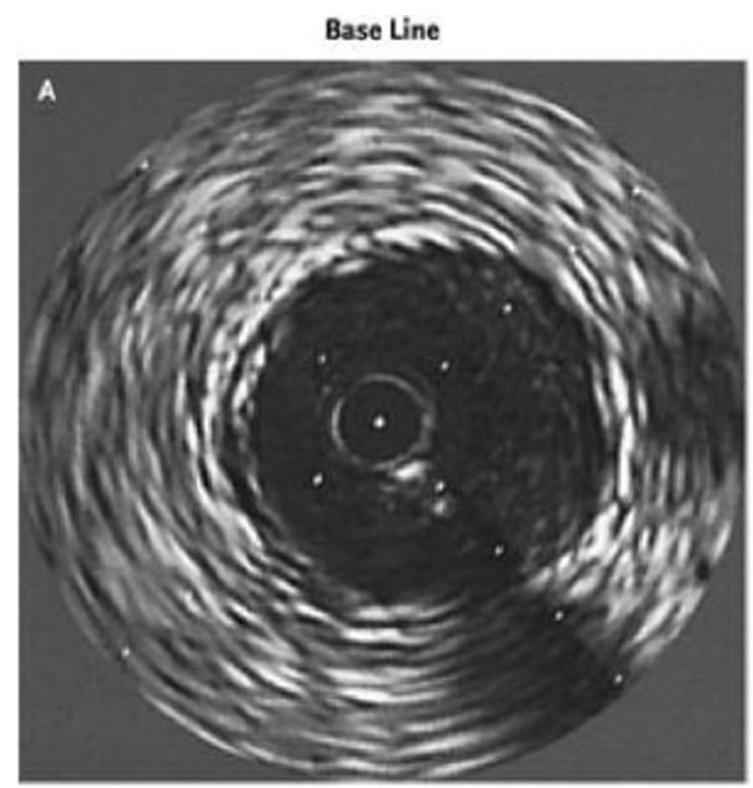

Maximal intimal thickness, $0.3 \mathrm{~mm}$

Fig. 3 An example of cardiac allograft vascuopathy. Note the change in maximal intimal thickness $>0.05 \mathrm{~mm}$ from the baseline IVUS study (Panel A) to the 12-month IVUS. (From Eisen HJ, Tuzcu EM, Dorent

renal function worsened when full-dose cyclosporine was used with the PSIs, triglycerides were elevated, and the IVUS population was a subset of the total study population, raising questions of unintentional bias. For example, patients with renal insufficiency might not have been referred for IVUS or even coronary angiography because of the risk that dye would pose to renal function. A final limitation of these studies was that the comparator drug was azathioprine which was the major anti-proliferative agent used at the time the study was performed, but that has since been almost completely supplanted by mycophenolic acid formulations such as mycophenolate mofetil (MMF). Future clinical trials would have to use these drugs as comparators.

\section{Proliferation Signal Inhibitors Compared to Mycophenolate Mofetil In De Novo Cardiac Transplant Recipients}

The original 650-patient, randomized trial of MMF compared to azathioprine showed improved survival and a reduction in the requirement of treatment for rejection in patients receiving study drug [16]. The IVUS and quantitative coronary angiography studies showed no difference in progression of CAV between the MMF and azathioprine groups. A more contemporary re-analysis of the IVUS results of this study showed a modest effect of MMF on progression of CAV compared to azathioprine [17, 18]. The azathioprine group had a greater of patients with baseline to first-year change in maximal intimal thickness (MIT) $\geq 0.3 \mathrm{~mm}$ ( $43 \%$ vs. $23 \%, P=0.005)$, a greater

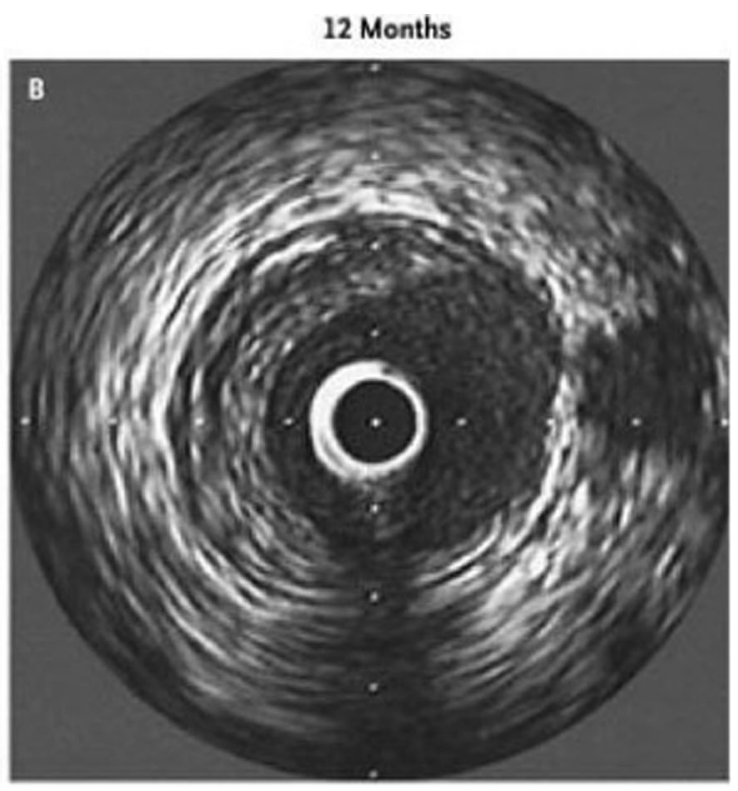

Maximal intimal thickness, $1.1 \mathrm{~mm}$

R, et al. Everolimus for the prevention of allograft rejection and vasculopathy in cardiac-transplant recipients. N Engl J Med. 2003; 349: 847858.)

decrease in the mean lumen area $(P=0.02)$ and a decrease in the mean vessel area (the area actually increased in the MMF group, $P=0.03$ ). There was no significant difference in the number of patients with baseline to first-year change in maximal intimal thickness (MIT) $\geq 0.5 \mathrm{~mm}(19 \%$ vs. $10 \%, P=$ $0.10)$ between the azathioprine and MMF groups.

In order to compare everolimus to the more contemporary clinical immunosuppression in which MMF is the preponderant anti-proliferative agent, more recent clinical trials have used this agent as a comparator. Also, a reduced cyclosporine dose and target trough level were used in the everolimus group to prevent the abnormal renal function that was seen in the earlier clinical trials. Lehmkuhl and colleagues randomized 176 patients from Europe, Brazil, and Israel to receive either everolimus (target trough level $3-8 \mathrm{ng} / \mathrm{mL}$ ) with reduced cyclosporine or MMF 3.0 gm daily with standard cyclosporine dose and trough target levels; both groups received corticosteroids and depending on the program, induction $[17,18]$. At 12 months, both groups had similar renal function as reflected by GFR (everolimus 68.7+/-27.7 and MMF 71.8+/$29.8 \mathrm{~mL} / \mathrm{min}$ ). Rates of acute cellular rejection of Grade $3 \mathrm{~A}$ (2R) rejection were not significantly different for everolimus $(22.8 \%)$ and MMF (29.8\%) at 12 months. As with the previous study, CMV infections were significantly less frequent in the everolimus group $(4.4 \%, P=0.01)$ than in the MMF group $(16.9 \%)$. This study demonstrated that everolimus and reduced cyclosporine dose and target trough levels could avoid the renal dysfunction seen in the earlier study.

Using these observations, 724 patients from 63 transplant centers on five continents were randomized in an open label 
study to either everolimus $1.5 \mathrm{mg}$ or $3.0 \mathrm{mg}$, both with reduced cyclosporine dose and target trough levels, or MMF $3.0 \mathrm{gm}$ with standard cyclosporine dose and trough levels [19•]. Both groups also received corticosteroids and induction therapy (thymoglobulin or basiliximab) if that was the center's standard practice. The primary efficacy endpoint was the composite of biopsy-proven ISHLT Grade 3A (2R) rejection, rejection with hemodynamic compromise, death, graft loss or retransplantation, or loss to follow-up. Everolimus was noninferior to MMF at 12 months for the composite endpoint and for each component of the endpoint. The primary safety endpoint was renal function at 12 months expressed as the eGFR using the MDRD approach. For this endpoint, eGFR was significantly worse for the everolimus $1.5 \mathrm{mg}$ group at 12 months $\left(59.4+/-22.8 \mathrm{~mL} / \mathrm{min} / 1.73 \mathrm{~m}^{2}\right)$ compared to MMF $\left(64.7+/-28.1 \mathrm{~mL} / \mathrm{min} / 1.73 \mathrm{~m}^{2}, P=0.009\right)$. Ten of the 63 centers did not achieve reduced target trough levels of cyclosporine in the everolimus groups, and if these centers were excluded (in a post hoc analysis), no significant difference was seen in the eGFR, demonstrating the importance of reducing cyclosporine dose to achieve the reduced target trough levels and thereby protect renal function.

The $3.0 \mathrm{mg}$ group had a higher mortality at 12 months than the other groups and enrollment in this group was ended by the DSMB. There was also a higher mortality rate at 12 months in the everolimus $1.5 \mathrm{mg}$ group ( $7.5 \%$ ) compared to the MMF group $(4.9 \%)$. The deaths in the everolimus group were primarily in patients who received everolimus and thymoglobulin; there were comparable numbers of deaths in everolimus $1.5 \mathrm{mg}$ and MMF patients who received no induction therapy of basiliximab. At 24 months, these findings demonstrate the risk of infection in patients who are overimmunosuppressed and have been observed in another clinical trial where the combination of daclizumab and thymoglobulin resulted in an increase in death from fungal infections [20]. At month 24, the mortality rates for the everolimus $1.5 \mathrm{mg}$ groups was $10 \%$ versus $9.2 \%$ for MMF.

There was no significantly increased rate of mediastinitis or wound infections in patients receiving everolimus compared to those receiving MMF. Pericardial effusions, including those requiring pericardial drainage, were more common in the everolimus $1.5 \mathrm{mg}$ group $(13.3 \%)$ compared to the MMF group $(4.1 \%)$ at 12 months. No deaths resulted from the pericardial effusions. As with previous studies, CMV infections were significantly less frequent in the everolimus $1.5 \mathrm{mg}$ group $(7.2 \%, P=0.001)$ compared to the MMF group $(19.4 \%)$. Bacterial infections were more common in the everolimus group (30.1\%) versus the MMF group (22.0\%).

An IVUS sub-study was performed to assess the effect of everolimus on progression of CAV as measured by IVUS compared to MMF $[17,18,19 \bullet]$. There was less progression of CAV as expressed by multiple parameters with everolimus compared to MMF. In particular, the mean change in MIT from baseline to the 1-year IVUS study for everolimus was $0.03+/-0.05(P<0.001)$ versus $0.07+/-0.11$ for the MMF groups. Volumetric parameters also showed less progression in the everolimus group than in the MMF group. The mean incidence of CAV which was defined as in the previous everolimus randomized clinical trial [13] was $12.5 \%(P=$ 0.018 ) for the everolimus group compared to $26.7 \%$ for MMF (Fig. 3). These findings were consistently seen even in high-risk groups such as diabetics, patients with hyperlipidemia and with pre-existing donor disease [21•] (Fig. 4).

From this study, it can be concluded that everolimus is superior to MMF for preventing CAV and CMV and that if care is taken to reduce cyclosporine exposure, the renal insufficiency seen with PSIs and full-dose CNIs can be avoided. Echocardiography should be performed in the first month post-transplant to identify those patients with significant pericardial effusions and concurrent use of everolimus, and thymoglobulin induction therapy should be avoided. Use of PSIs in patients with underlying significant renal insufficiency at the time of transplant or with infected ventricular assist devices should also be avoided.

An alternative approach for implementing therapy with everolimus to ameliorate $\mathrm{CAV}$ while minimizing adverse renal effects is to eliminate cyclosporine in the early posttransplant period [22]. Arora and colleagues randomized 115 patients at five Scandinavian transplant centers to receive standard cyclosporine/MMF/corticosteroid therapy or everolimus in the first few days after transplant with weaning of cyclosporine at weeks $7-11$ post-transplant. IVUS was performed at baseline and 12 months after transplant. Fortyseven patients in the everolimus group and 48 in the standard care group have baseline and 12-month IVUS studies. Change in MIT and volumetric measures such as percent atheroma (PAV) and total atheroma volumes (TAV) were assessed. The everolimus group had significantly reduced $\mathrm{CAV}$ progression as compared to the CNI group [ $\triangle \mathrm{MIT} 0.03 \pm 0.06$ and $0.08 \pm$ $0.12 \mathrm{~mm}, \triangle \mathrm{PAV} 1.3 \pm 2.3$ and $4.2 \pm 5.0 \%, \Delta \mathrm{TAV} 1.1 \pm$ $19.2 \mathrm{~mm}^{3}$ and $13.8 \pm 28.0 \mathrm{~mm}^{3}$ (all $P$-values $\leq 0.01$ )]. The number of patients with $\geq 2 \mathrm{R}$ rejection was $20(43 \%)$ in the everolimus group compared to seven $(15 \%)$ in the cyclosporine group $(P<0.01)$. Fifteen percent of everolimus patients were converted to a regimen of low-dose CNI and everolimus due to recurrent rejections. Renal function and survival were similar between the two groups. This study illustrates that early elimination of CNI in the presence of PSIs is possible and feasible in cardiac transplant recipients with preservation of the beneficial effects of PSIs on CAV progression and with preservation of renal function, which is a challenge (but clearly can be done) in regimens of long-term concomitant PSI and CNI use. The only caveat with the elimination strategy is the increased risk of cellular rejection. 
Fig. 4 Subgroup analysis of change in Mean MIT from baseline to 12-month IVUS study in everolimus versus MMF clinical trial (From Kobashigawa JA, Pauly DF, Starling RC, Eisen $\mathrm{H}$, et al. A2310 IVUS Substudy Investigators.Cardiac allograft vasculopathy by intravascular ultrasound in heart transplant patients: substudy from the Everolimus versus mycophenolate mofetil randomized, multicenter trial. JACC Heart Fail. 2013;1:389-99.)

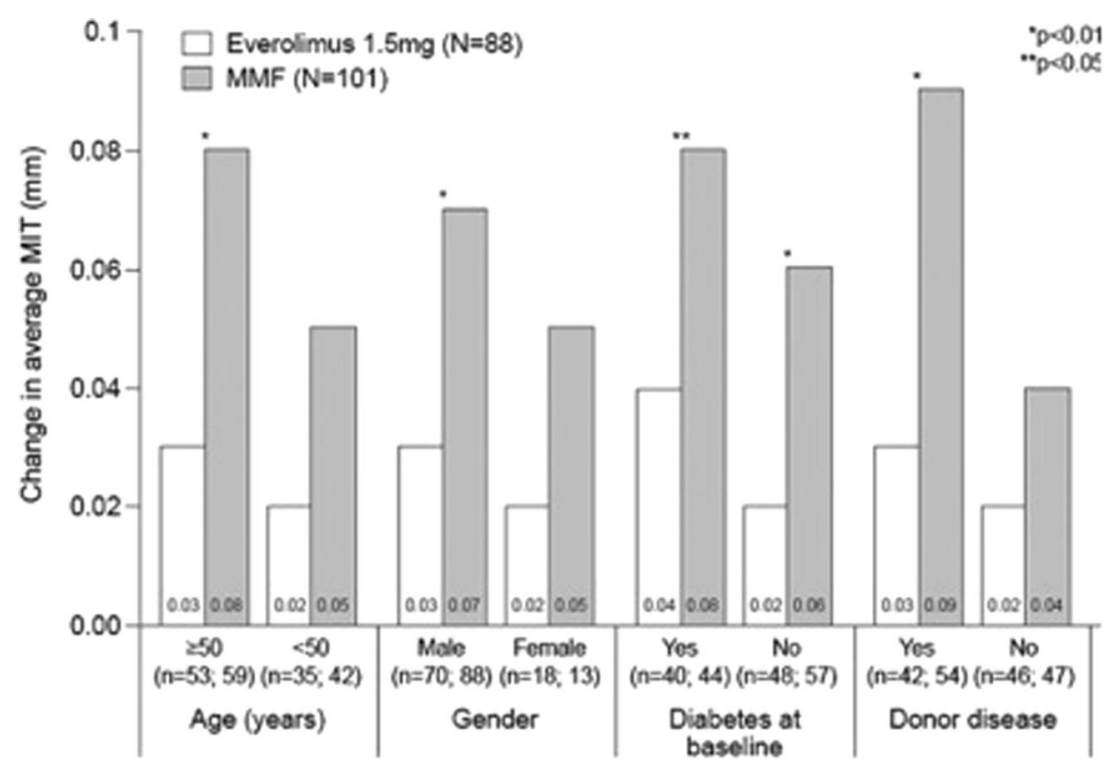

\section{Use of PSIs In De Novo Cardiac Transplant Recipients Outside of Clincal Trials}

Kuppahally and colleagues introduced the use of sirolimus in de novo transplant patients because of its potential salutary effects on CAV and rejection [23]. They retrospectively compared the outcomes of 48 patients who received sirolimus in addition to CNI and corticosteroids immediately after transplant to a historical control population who received MMF, $\mathrm{CNI}$, and steroids. The incidence of any post-surgical wound complication (52\% vs. $28 \%, P=0.019$ ) and deep surgical wound complication ( $35 \%$ vs. $13 \%, P=0.012$ ) was significantly higher in the sirolimus group than in MMF one. Patients on sirolimus were also more likely to have symptomatic pleural $(P=0.035)$ and large pericardial effusions $(P=$ 0.033 ) requiring intervention. This experience led to the abandonment of sirolimus as de novo therapy for heart transplant recipients. Of note, the increased wound complications and large pleural effusions were not noted in the everolimusMMF clinical trial although large pericardial effusions were observed.

\section{Use PSIs and Tacrolimus In De Novo Cardiac Transplant Recipients}

Sirolimus plus tacrolimus was compared to tacrolimus plus MMF or cyclosporine plus MMF in a three-arm clinical trial [24]. All patients received corticosteroids as well. The primary endpoint of this study was incidence of $\geq 3 \mathrm{~A}$ (2R) rejection or rejection with hemodynamic compromise. The tacrolimusMMF arm was superior because it had fewer rejections at 12 months compared to the cyclosporine-MMF arm (23.4\% vs. $36.8 \%, P=0.029)$. The incidence of any treated rejection was significantly higher in the cyclosporine-MMF arm (59\%, $P=0.001)$ compared to both tacrolimus-sirolimus ( $35 \%$ ) and tacrolimus-MMF (42\%). The tacrolimus-sirolimus arm had higher median serum creatinine levels $(1.5 \mathrm{mg} / \mathrm{dL}, P=0.032)$ compared to the tacrolimus-MMF arm $(1.3 \mathrm{mg} / \mathrm{dL})$. Impaired wound healing was more frequent in with tacrolimus-sirolmus as were fungal infections while viral infections were less common. The authors concluded that tacrolimus-MMF offered the most advantages in terms of preventing rejection with fewer side effects.

\section{PSIs for the Prevention pf CAV in Maintenance Cardiac Transplant Recipients}

Given the complexity of concomitantly initiating therapy with PSIs and CNIs in the early post-transplant period, there is considerable interest in developing immunosuppressive protocols that commence the use of PSIs months or more after transplant. Topilsky converted 45 cardiac transplant recipients to sirolimus a mean of 1.2 years after transplant (range $0.2-$ 4.0 years) with subsequent withdrawal of CNI inhibitors and compared progression of CAV defined by IVUS with a group of 58 patients who were maintained on CNI inhibitors [25•]. Three-dimensional IVUS was performed at baseline and on average 3.1 years (range $1.3-4.6$ years) later. Plaque index progression (plaque volume/vessel volume) was attenuated in the sirolimus group $(0.7 \pm 10.5 \%$ versus $9.3 \pm 10.8 \%$; $P=$ $0.0003)$ due to reduced plaque volume in patients converted to sirolimus early ( $<2$ years) after transplantation $(P=0.05)$, and improved positive vascular remodeling $(P=0.01)$ in patients analyzed later ( $>2$ years) after transplantation. Five-year survival was $97.4+/-1.8 \%$ for patients converted to sirolimus compared to $81.8+/-4.9 \%$ for patients receiving CNI-based 
therapy $(P=0.006)$. Freedom from MACE was $93.6+/-3.2 \%$ for patients converted to sirolimus versus $76.9+/-5.5 \%$ for those managed with $\mathrm{CNI}(P=0.002)$. This study suggests that PSIs initiated later after transplant and not in de novo patients can attenuate $\mathrm{CAV}$ and as a result, favorably affect survival and the development of MACE. A major limitation of this study is that it was not a randomized, controlled trial.

The NOCTET study was designed to study the effects of changing immunosuppression from $\mathrm{CNI}$ to everolimus and reduced-dose $\mathrm{CNI}$ in cardiac transplant recipients with mild to moderate renal dysfunction [26]. This study included patients who were further out (mean 5.8+/-4.3 years) from transplant than the Topilsky study [24], and progression of CAV was assessed using IVUS. Patients on CNI without everolimus were studied as controls. No attenuation in CAV was seen in the everolimus-low-dose CNI group compared to the CNI group, suggesting that addition of PSI therapy should be done earlier (perhaps less than 2 years post-transplant). Interestingly, background immunosuppression had an important influence on CAV progression; patients receiving everolimus and azathioprine had attenuated progression of CAV, whereas those receiving everolimus and MMF had accelerated CAV progression.

Addition of PSIs and reduction or elimination of CNIs to ameliorate post-transplant renal dysfunction is a common clinical strategy. Two studies have examined this approach. The NOCTET study mentioned above was an open-label study of 245 Scandinavian thoracic transplant patients more than 1 year post-transplant with mild to moderate renal dysfunction [27]. Patients were randomized to remain on standard CNI regimen or to switch to a regimen of everolimus and reduced CNI exposure. At 24 months, mean GFR increased by $3.2 \pm 12.3 \mathrm{~mL} / \mathrm{min}$ from the point of randomization in everolimus-treated patients and decreased by $2.4 \pm 9.0 \mathrm{~mL} /$ $\min$ in controls $(P<0.001)$. There was no difference in rates of biopsy-proven rejection or adverse events. Analysis of benefit for conversion to the everolimus regimen based on degree of renal dysfunction demonstrated that improved GFR was seen in patients with baseline GFRs of 20 to $59 \mathrm{~mL} / \mathrm{min} /$ $1.73 \mathrm{~m}^{2}$ but not in patients with GFRs of 60 or greater and also not in patients who underwent conversion more than 4 years post-transplant [28•].

Zuckermann and colleagues randomized 116 patients $1-$ 8 years post-transplant with GFRs of $40-90 \mathrm{~mL} / \mathrm{min} / 1.73 \mathrm{~m}^{2}$ either to remain on CNI-based therapy $(n=59)$ or receive sirolimus with withdrawal of CNI [29•]. At 1 year, the GFR was significantly higher with sirolimus versus $\mathrm{CNI}(+3.0$ vs. $1.4 \mathrm{~mL} / \mathrm{min} / 1.73 \mathrm{~m}^{2}$, respectively; $P=0.004$ ) for intention-totreat analysis and for on-therapy analysis $(+4.7$ and -2.1 , respectively; $P<0.001)$. Acute rejection was more common with sirolimus as was the rate of therapy discontinuation ( $33.3 \%$ for sirolimus vs. $0 \%$ for CNI, $P<0.001$ ). Diarrhea, rash, and infections were all more common in the sirolimus group. These results would indicate that although conversion to sirolimus therapy can improve renal function, it comes with the price of side effects and increased risk of rejection.

\section{Use of PSIs to Halt the Progression of Established CAV}

Once CAV is identified, the tendency is to start PSIs because of their anti-proliferative effects and their beneficial role in blunting the progression of CAV in de novo patients. Patients with established CAV represent a different population. Mancini and colleagues randomized 46 patients with severe CAV who were on average $4.3+/-2.3$ years post-transplant to have sirolimus added to their regimen $(n=22)$ or continue on their present regimen [30]. Progression of disease was measured angiographically using a score, and outcomes such as MI, death, and need for PCI or CABG was assessed. Patients in the sirolimus group had no progression of CAV angiographically, and only three patients in the sirolimus group had adverse events compared to 14 in the control group. This study, although small, would suggest that there is a benefit to adding PSIs to the immunosuppressive regimen of patients with established CAV. The Spanish EVEROSTAT study enrolled patients with angiographic evidence of CAV and randomly assigned them to stay on their CNI regimen $(n=15)$ or to switch to everolimus and reduced-dose CNI $(n=35)[31]$. There was no difference in MACE between the two groups, but in the IVUS sub-study, everolimus patients $(n=19)$ had less progression or even regression of CAV compared to the control group $(n=8)$ [32]. Both of these studies should serve as pilots for a larger randomized trial of the effect of everolimus on established CAV.

\section{Use of PSIs in Cardiac Transplant Recipients with Malignancies}

There are no randomized studies of the effects of PSIs on cardiac transplant recipients with malignancies. A retrospective study of renal transplant recipients from the SRTR showed that those receiving PSIs with or without CNIs were less likely to develop malignancies than those receiving CNIs without PSIs [33]. Whether PSI therapy can affect the outcomes of established malignancies in cardiac transplant recipients is unknown although they are used as adjunctive therapy in patients with breast and renal cell carcinoma and other malignancies [34].

\section{Conclusion}

PSIs are a group of immunosuppressive agents with the unique ability to inhibit cell proliferation, including vascular 
smooth muscle cells. They, therefore, offer the potential to attenuate the progression of CAV and perhaps favorably alter post-transplant outcomes as a result. They have many side effects and interactions with other drugs such as cyclosporine, which complicates their use. Development of immunosuppressive regimens which minimize these side effects while preserving their beneficial effects on CAV are crucial if they are to be widely used and to reach their potential.

\section{Compliance with Ethics Guidelines}

Conflict of Interest $\mathrm{H}$ Eisen declares no conflicts of interest.

Human and Animal Rights and Informed Consent All studies by the author involving animal and/or human subjects were performed after approval by the appropriate institutional review boards. When required, written informed consent was obtained from all participants.

\section{References}

Papers of particular interest, published recently, have been highlighted as:

- Of importance

1. Lund LH, Edwards LB, Kucheryavaya AY, et al. The Registry of the International Society for Heart and Lung Transplantation: Thirtieth Official Adult Heart Transplant Report-2013; focus theme: age. International Society for Heart and Lung Transplantation. J Heart Lung Transplant. 2013;32:951-64.

2. Kobashigawa JA, Katznelson S, Laks H, et al. Effect of pravastatin on outcomes after cardiac transplantation. N Engl J Med. 1995;333: $621-7$.

3. Wenke K, Meiser B, Thiery J, et al. Simvastatin initiated early after heart transplantation: 8-year prospective experience. Circulation. 2003;107:93-7.

4. Meric-Bernstam F, Gonzalez-Angulo AM. Targeting the mTOR Signaling Network for Cancer Therapy. J Clin Oncol. 2009;27: 2278-87.

5. Brown EJ, Albers MW, Shin TB, et al. A mammalian protein targeted by G1-arresting rapamycin-receptor complex. Nature. 1994;369:756-8.

6. Schmid C, Heemann U, Azuma H, et al. Rapamycin inhibits transplant vasculopathy in long-surviving rat heart allografts. Transplantation. 1995;60:729-33.

7. Gregory C, Huang X, Pratt RE, et al. Treatment with rapamycin and mycophenolic acid reduces arterial intimal thickening produced by mechanical injury and allows endothelial replacement. Transplantation. 1995;59:655-61.

8. Morice MC, Serruys PW, Sousa JE, et al. A randomized comparison of a sirolimus-eluting stent with a standard stent for coronary revascularisation. N Engl J Med. 2002;346:1773-80.

9. Moses JW, Leon MB, Popma JJ, SIRIUS Investigators, et al. Sirolimus-eluting stents versus standard stents in patients with stenosis in a native coronary artery. N Engl J Med. 2003;349: 1315-23.

10. Byrne RA, Kastrati A, Massberg S, ISAR-TEST 4 Investigators, et al. Biodegradable polymer versus permanent polymer drugeluting stents and everolimus- versus sirolimus-eluting stents in patients with coronary artery disease: 3 -year outcomes from a randomized clinical trial. J Am Coll Cardiol. 2011;58:1325-31.

11. Farb A, Burke AP, Kolodgie FD, Virmani R. Pathological mechanisms of fatal late coronary stent thrombosis in humans. Circulation. 2003;108:1701-6.

12. Keogh A, Richardson M, Ruygrok P, et al. Sirolimus in de novo heart transplant recipients reduces acute rejection and prevents coronary artery disease at 2 years: a randomized clinical trial. Circulation. 2004;110:2694-700.

13. Eisen HJ, Tuzcu EM, Dorent R, et al. Everolimus for the prevention of allograft rejection and vasculopathy in cardiac-transplant recipients. N Engl J Med. 2003;349:847-58.

14. Starling RC, Hare JM, Hauptman P, et al. Therapeutic drug monitoring for everolimus in heart transplant recipients based on exposure-effect modeling. Am J Transplant. 2004;4:2126-31.

15. Eisen $\mathrm{H}$. Long-term cardiovascular risk in transplantation-insights from the use of everolimus in heart transplantation. Nephrol Dial Transplant. 2006;21 Suppl 3:iii9-13.

16. Kobashigawa JA, Miller L, Renlund D, et al. A randomized activecontrolled trial of mycophenolate mofetil in heart transplant recipients. Mycophenolate Mofetil Investigators. Transplantation. 1998;66:507-15.

17. Kobashigawa JA, Tobis JM, Mentzer RM, et al. Mycophenolate mofetil reduces intimal thickness by intravascular ultrasound after heart transplant: reanalysis of the multicenter trial. Am J Transplant. 2006;6:993-7.

18. Lehmkuhl HB, Arizon J, Viganò M, 2411 Study Investigators, et al. Everolimus with reduced cyclosporine versus MMF with standard cyclosporine in de novo heart transplant recipients. Transplantation. 2009;88:115-22.

19. Eisen HJ, Kobashigawa J, Starling RC, et al. Everolimus versus mycophenolate mofetil in heart transplantation: a randomized, multicenter trial. Am J Transplant. 2013;13:1203-16. The largest clinical trial in cardiac transplantation. Demonstrates efficacy of everolimus in ameliorating CAV compared to MMF and highlights complexities and pitfalls of using this medication.

20. Hershberger RE, Starling RC, Eisen HJ, et al. Daclizumab to prevent rejection after cardiac transplantation. N Engl J Med. 2005;352:2705-13.

21. Kobashigawa JA, Pauly DF, Starling RC, Eisen H, A2310 IVUS Substudy Investigators, et al. Cardiac allograft vasculopathy by intravascular ultrasound in heart transplant patients: substudy from the Everolimus versus mycophenolate mofetil randomized, multicenter trial. JACC Heart Fail. 2013;1:389-99. Further illumintaes beneficial effects of everolimus compared to MMF for progression of CAV.

22. Arora S, Andersson F, Gustaffson H, et al. Everolimus Initiation With Calcineurin Inhibitor Withdrawal Reduces Allograft Vasculopathy in De-Novo Heart Transplant Recipients. J Heart Lung Transplant. 2014;33:S33-4. abstract.

23. Kuppahally S, Al-Khaldi A, Weisshaar D, et al. Wound healing complications with de novo sirolimus versus mycophenolate mofetil-based regimen in cardiac transplant recipients. Am J Transplant. 2006;6:986-92.

24. Kobashigawa JA, Miller LW, Russell SD, et al. Tacrolimus with mycophenolate mofetil (MMF) or sirolimus vs. cyclosporine with MMF in cardiac transplant patients: 1-year report. Am J Transplant. 2006;6:1377-86.

25. Topilsky Y, Hasin T, Raichlin E, et al. Sirolimus as primary immunosuppression attenuates allograft vasculopathy with improved late survival and decreased cardiac events after cardiac transplantation. Circulation. 2012;125:708-20. Demonstrates that initiation of sirolimus after the first year post-transplant can have a beneficial effect on the progression of CAV.

26. Arora S, Ueland T, Wennerblom B, et al. Effect of everolimus introduction on cardiac allograft vasculopathy-results of a randomized, multicenter trial. Transplantation. 2011;92:235-43. 
27. Gullestad L, Mortensen SA, Eiskjær H, et al. Two-year outcomes in thoracic transplant recipients after conversion to everolimus with reduced calcineurin inhibitor within a multicenter, open-label, randomized trial. Transplantation. 2010;90:1581-9.

28. Arora S, Gude E, Sigurdardottir V, et al. Improvement in renal function after everolimus introduction and calcineurin inhibitor reduction in maintenance thoracic transplant recipients: the significance of baseline glomerular filtration rate. J Heart Lung Transplant. 2012;31:259-65. This paper shows that the introduction of everolimus to the immunosuppressive regimen of cardiac transplant patients improves renal function in thos with moderate to severe but not mild renal dysfunction from CNIs.

29. Zuckermann A, Keogh A, Crespo-Leiro MG, et al. Randomized controlled trial of sirolimus conversion in cardiac transplant recipients with renal insufficiency. Am J Transplant. 2012;12:2487-97. This study also demonstrates the benefit of conversion to PSIs on renal function but highlights the risk of rejection and side effects from PSIs.
30. Mancini D, Pinney S, Burkhoff D, et al. Use of rapamycin slows progression of cardiac transplantation vasculopathy. Circulation. 2003;108:48-53.

31. Segovia J, Fernandez-Yanez J, Gonzalez-Vilchez FJ, et al. Clinical Effects of Introducing Everolimus in the Immunosuppressive Regimen of Patients with Established Cardiac Allograft Vasculopathy (CAV): Efficacy Analysis of a Randomized, Multicenter Study. J Heart Lung Transplant. 2012;31:S121. abstract.

32. Segovia J, Gomez-Bueno M, Goicolea J, et al. Effect of Everolimus in Patients with Established Cardiac Allograft Vasculopathy: Results of a Randomized, Multicenter Intravascular Ultrasound Study. J Heart Lung Transplant. 2012;31:S77.

33. Kauffman HM, Cherikh WS, Cheng Y, et al. Maintenance immunosuppression with target-of-rapamycin inhibitors is associated with a reduced incidence of de novo malignancies. Transplantation. 2005;80:883-9.

34. Baselga J, Campone M, Piccart M, et al. Everolimus in postmenopausal hormone-receptor-positive advanced breast cancer. N Engl J Med. 2012;366:520-9. 